5. Bearing on the Nature of the Theory of Deduction. The fact that proposition (viii) cannot be derived from the theory of deduction has an important bearing on the nature of that theory. The theory of deduction has been designed as "the calculus of propositions." Proposition (viii) is a well known proposition in the classic logic of propositions; the theory cannot yield this proposition; and so the theory cannot serve as "the calculus of propositions."

The University of California

\title{
A SUFFICIENT CONDITION FOR THE EXISTENCE OF A DOUBLE LIMIT
}

BY J. A. CLARKSON

In the elementary theory of limits it is often emphasized that the existence of a unique limit for a single-valued function $f(x, y)$ as the point $P(x, y)$ approaches $Q(a, b)$ along every straight line through $Q$ does not imply the existence of the double limit

$$
\lim _{\substack{x \rightarrow a \\ y \rightarrow b}} f(x, y) .
$$

As early as 1873 Thomae* gave an example to illustrate this fact.

The question then naturally arises: Is the existence of a unique limit as $P$ approaches $Q$ along some more extensive class of curves sufficient to insure the existence of (1)? This question is immediately answered by the following theorem.

Theorem. If $f(x, y)$ has a unique limit $L$ as $P(x, y)$ approaches $Q(a, b)$ along every curve having a tangent at $Q$, the double limit (1) exists.

Proof. Suppose, if possible, that it does not. Then there exists an $\epsilon>0$ such that in any circle about $Q$ there are points $p$ for which

$$
|f(p)-L|>\epsilon
$$

We denote by $E$ the set of all such points.

* J. Thomae, Abriss einer Theorie der complexen Functionen, 2d ed., Halle, 1873, p. 15. 
Let any circle $C$ with $Q$ as center be divided into quadrants; in at least one of these quadrants, considered as a closed region, the points of $E$ have $Q$ as a limit point. Let $p_{1}$ be any point of $E$ in such a quadrant, and $a_{1}$ the arc of $C$ bounding this quadrant.

Consider a second concentric circle with radius equal to half the length $Q p_{1}$, and the radius bisecting $a_{1}$. Let $p_{2}$ be any point of $E$ in one of the inner octants in which the points of $E$ have $Q$ as a limit point, and $a_{2}$ the corresponding half of $a_{1}$. This process may be continued indefinitely. As $n$ increases without limit, $p_{n}$ approaches $Q$, and the sequence of $\operatorname{arcs} a_{n}$ defines a point $R$ on the circumference.

Consider a curve $K$ passing through the points $p_{1}, p_{2}, p_{3}, \ldots$ in succession in such a manner that between $p_{n}$ and $p_{n+1}$, $(n=1,2,3, \cdots), K$ lies within the $n$th sector. Now if $P$ approaches $Q$ on $K$, the secant $Q P$ has the limiting direction $Q R$, and $f(x, y)$ must approach the limit $L$. But as the points $p_{n}$ on $K$ have $Q$ as a limit point, and (2) is satisfied for each $p_{n}$, the contradiction is apparent, and the proof is complete.

It may be noted that the hypothesis of the theorem can be weakened, since there will certainly exist a curve through the points $p_{1}, p_{2}, p_{3}, \cdots$ which possesses a tangent at every point. Moreover, it is clear that if a limit exists along each curve through $Q$ having a tangent at every point, the limit must be the same along all such curves having the same tangent at $Q$. A similar theorem can be proved in which the class of all curves with tangents at $Q$ is replaced by the class of all curves which do not possess tangents at $Q$.

BROWN UNIVERSITY 